\title{
Hydrolytic Enzymes as (Bio)-Logic for Wireless and Chipless Biosensors
}

Nigel F. Reuel ${ }^{1 *}$, Joseph McAuliffe ${ }^{2}$, Gregory A. Becht ${ }^{1}$, Mehrdad Mehdizadeh, ${ }^{3}$ Jeffrey Munos ${ }^{2}$, RuPing Wang $^{1}$, and William J. Delaney ${ }^{1}$

1. Central Research and Development - Materials Science Division - E. I. du Pont de Nemours and Company

2. Industrial Biosciences - E. I. du Pont de Nemours and Company

3. Engineering Technologies - E. I. du Pont de Nemours and Company

*To whom correspondence should be directed - nigel.f.reuel@dupont.com

\section{Supporting Information Table of Contents}

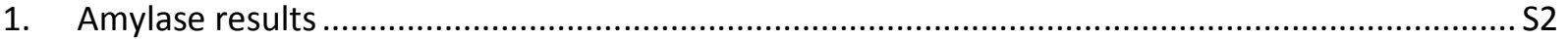

2. Digestion of $\alpha(1,3)$ Polysaccharide Film with Mutanase .......................................................... 44

3. S21 VNA Setup and Matlab Code for Copper Mountain DAQ ...................................................... S6

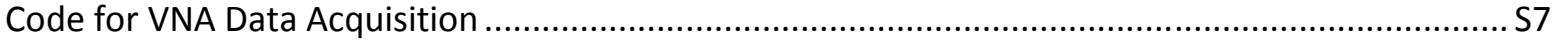

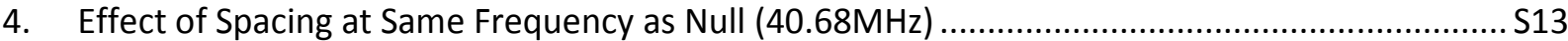

5. Response curves from the other divalent cations ................................................................ S14

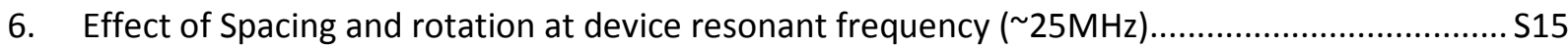

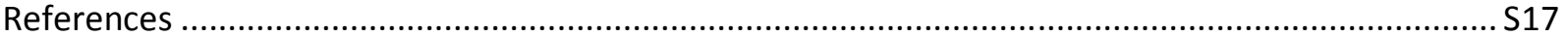




\section{Amylase results}

Amylase requires tightly bound calcium to remain active. Studies have shown methods for removing calcium to form apo-amylase by gentle dialysis ${ }^{1}$. Using ICP-MS we determined 48 hours in $\mathrm{pH} 6.9$ with $0.01 \mathrm{M}$ EDTA or EGTA was sufficient to lower the calcium content $5 x$ of amylase ( $\alpha$-Amylase from Bacillus subtilis, Sigma 10069) but had a limit of 1:2 calcium to amylase molar ratio (Fig S1). Using a standard starch activity assay (observing the level of digestion product maltose as it reacts with 3,5dinitrosalicylic acid stabilized with potassium sodium tartrate (Rochelle salt) to read the absorbance at $540 \mathrm{~nm}$ ) we find that the apo-amylase is not controlled by calcium concentration in solution (Fig S2) but is modulated by solution $\mathrm{pH}$ (Fig S3). Processing a freestanding, thin film of uniform thickness from starch also proved difficult.

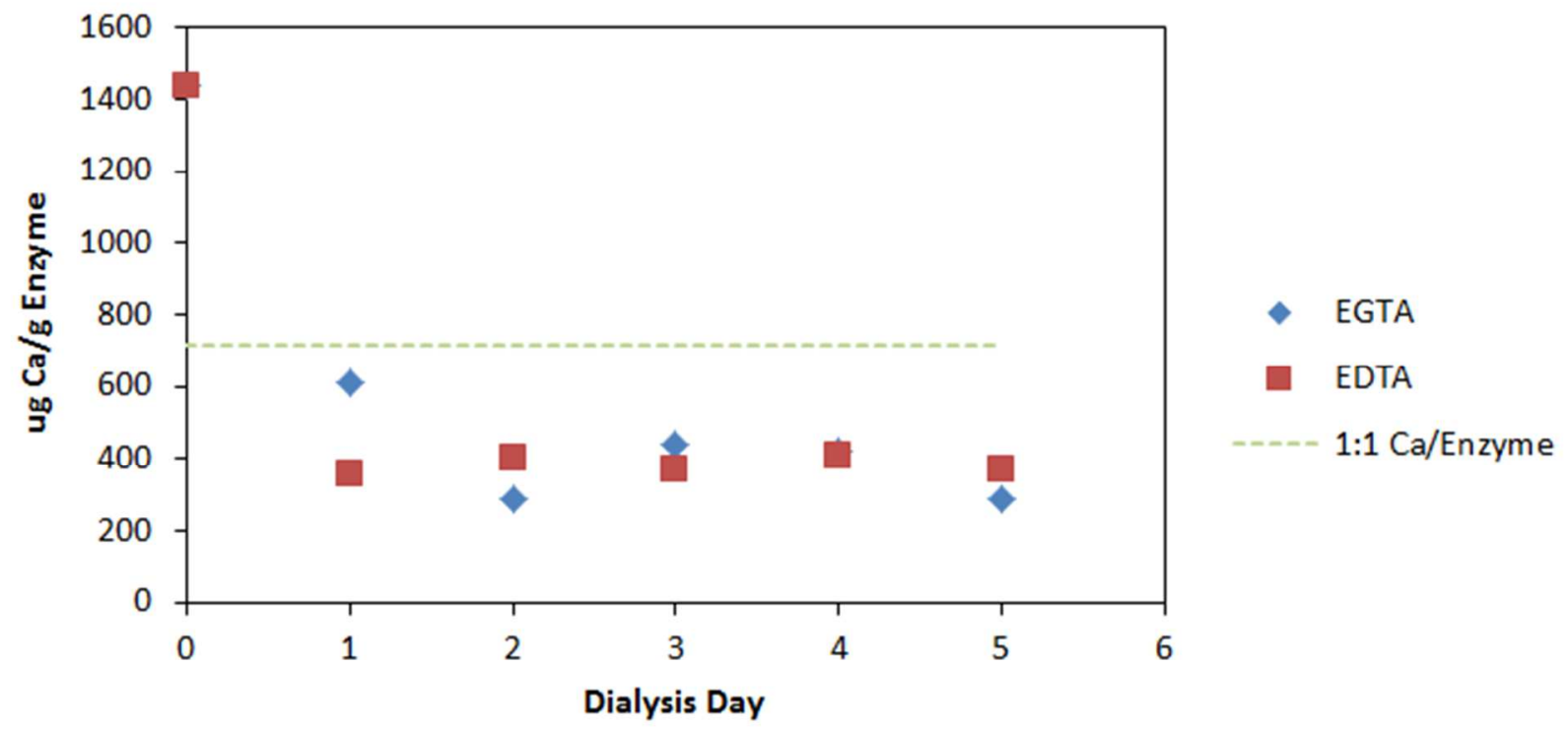

Figure S1 - ICP-MS results of dialysis treated amylase to determine calcium content. 


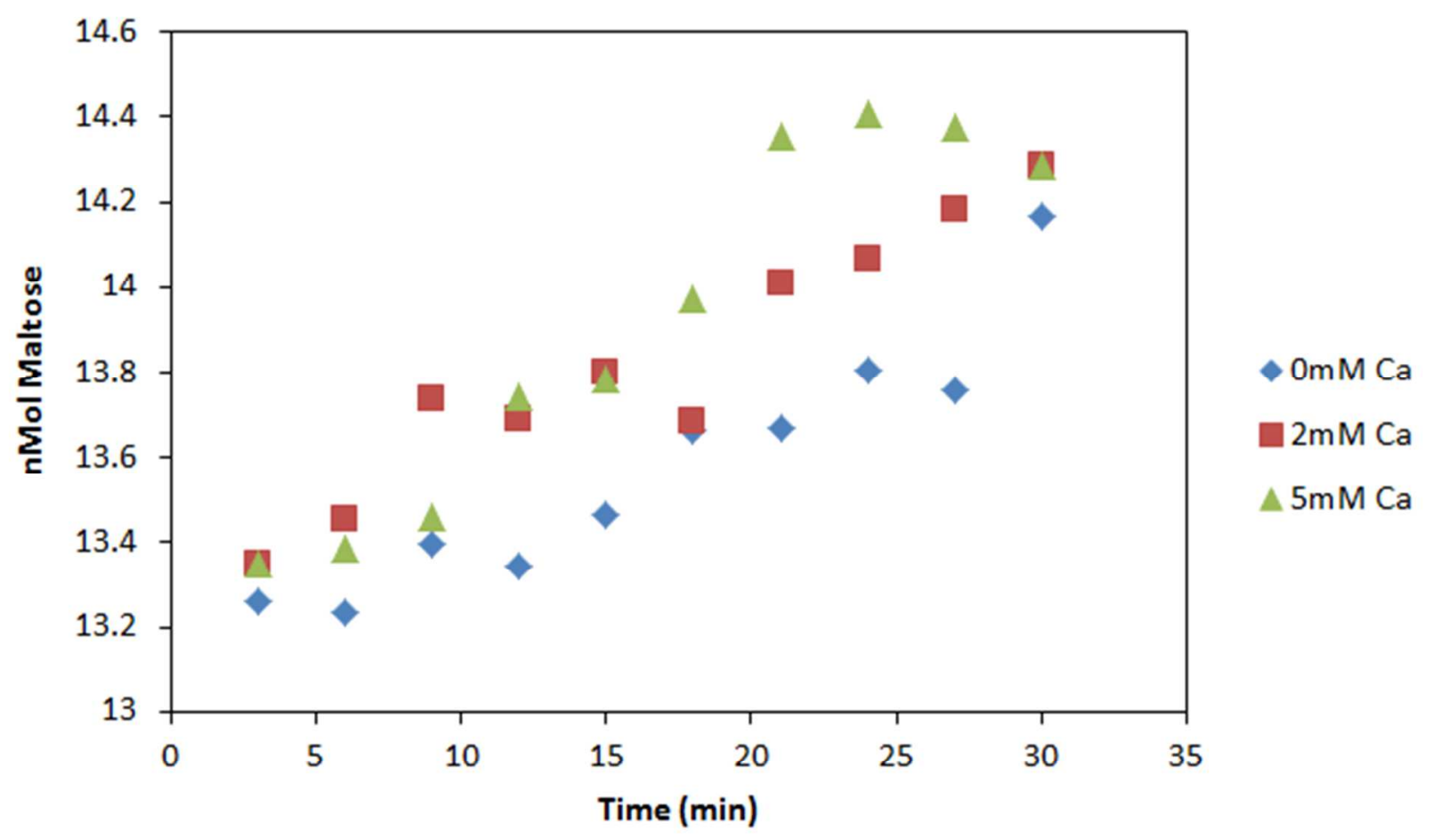

Figure S2 - Effect of calcium concentration on activity level of dialysis treated (48 h in 0.01 M EDTA) amylase at $0.5 \mu \mathrm{g} / \mathrm{ml}$.

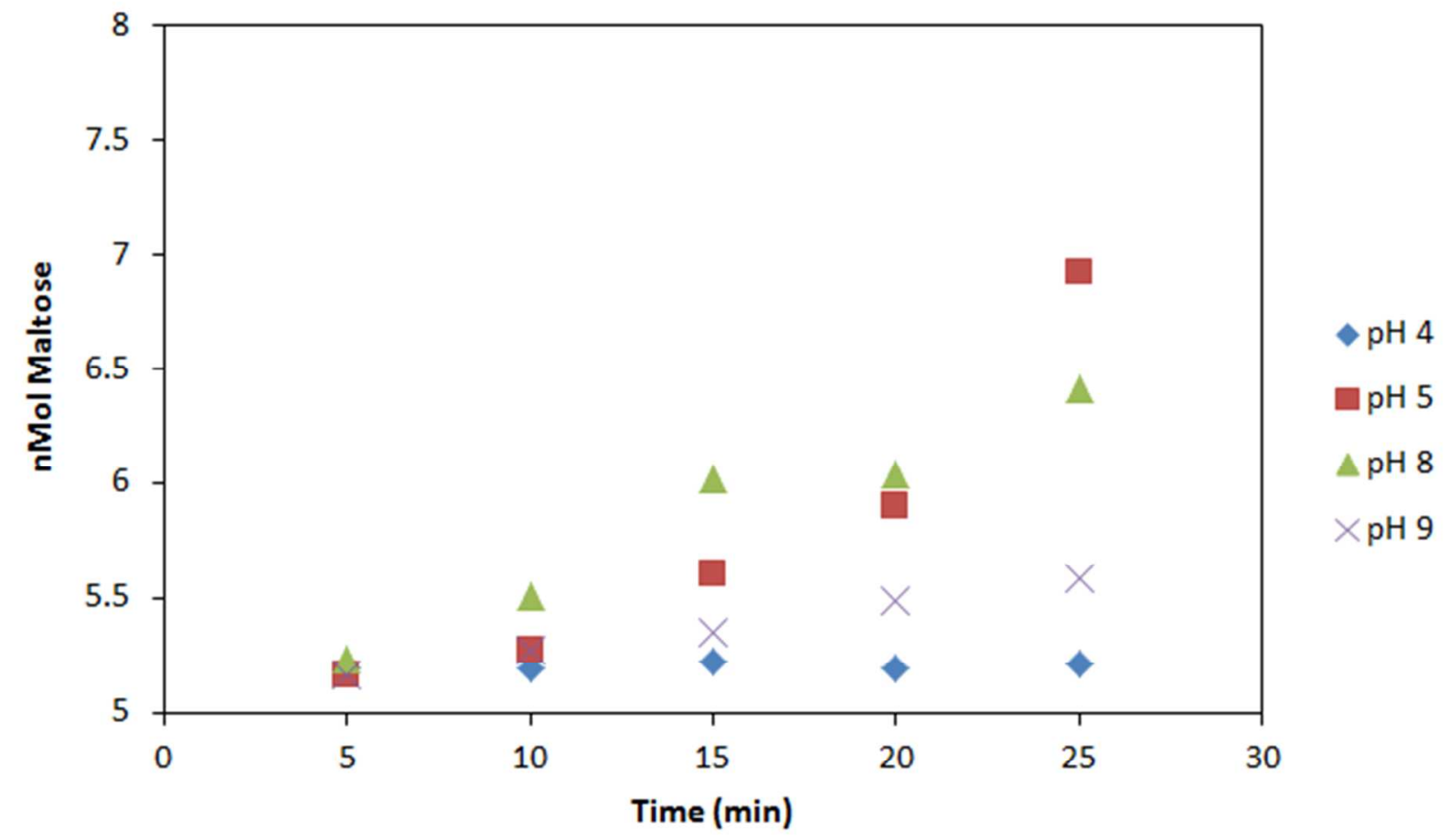

Figure S3 - Effect of pH on amylase activity. 


\section{Digestion of $\alpha(1,3)$ Polysaccharide Film with Mutanase}

$\alpha(1,3)$ polysaccharide film is another substrate candidate for a bio-based dielectric that can be digested by a mutanase enzyme ${ }^{2}$. A more hydrophobic $\alpha(1,3)$ polysaccharide film triacetate ester ${ }^{3}$ film (GTA) was tested in addition to a pure $\alpha(1,3)$ polysaccharide film ${ }^{4}$ film (GF). GTA showed little degradation after $24 \mathrm{~h}$ exposure to $1 \mathrm{mg} / \mathrm{ml}$ mutanase vs. GF as shown by micrographs (Fig S4) and absorbance of digested product, maltose (Fig S5). Testing the change in capacitance of the material also showed that GTA did not change after enzyme exposure, whereas GF modulated from 10 to 60 pF (Fig S6). However, we also found that mutanase could not be externally modified by cofactors or $\mathrm{pH}$ (demonstrated same activity over wide $\mathrm{pH}$ range (Fig S7).
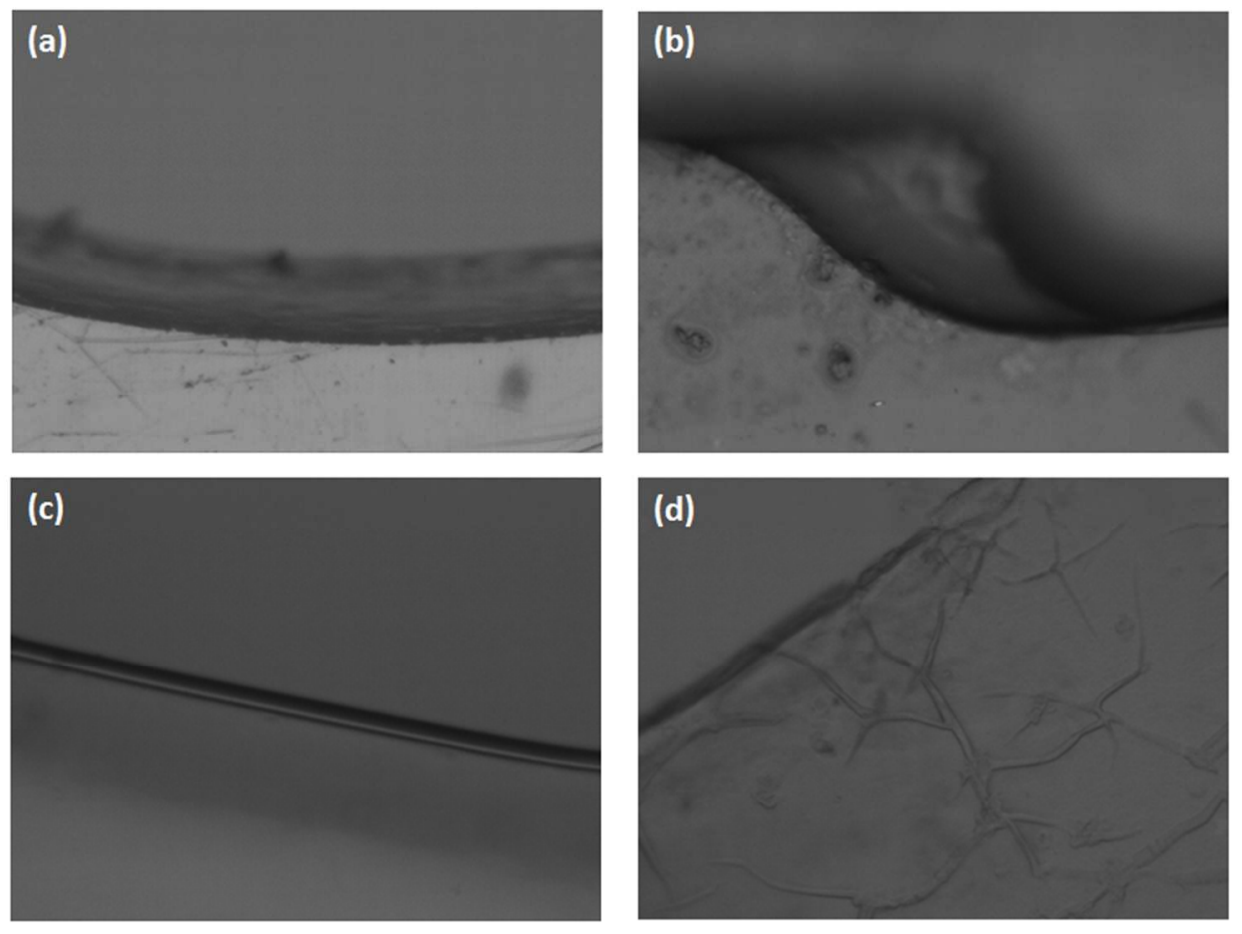

Figure S4 - Micrographs showing $\alpha(1,3)$ polysaccharide triacetate film before (a) and after (b) $24 \mathrm{~h}$ exposure to $1 \mathrm{mg} / \mathrm{ml}$ mutanase in contrast to pure $\alpha(1,3)$ polysaccharide film before (c) and (d) after the same enzyme exposure. 


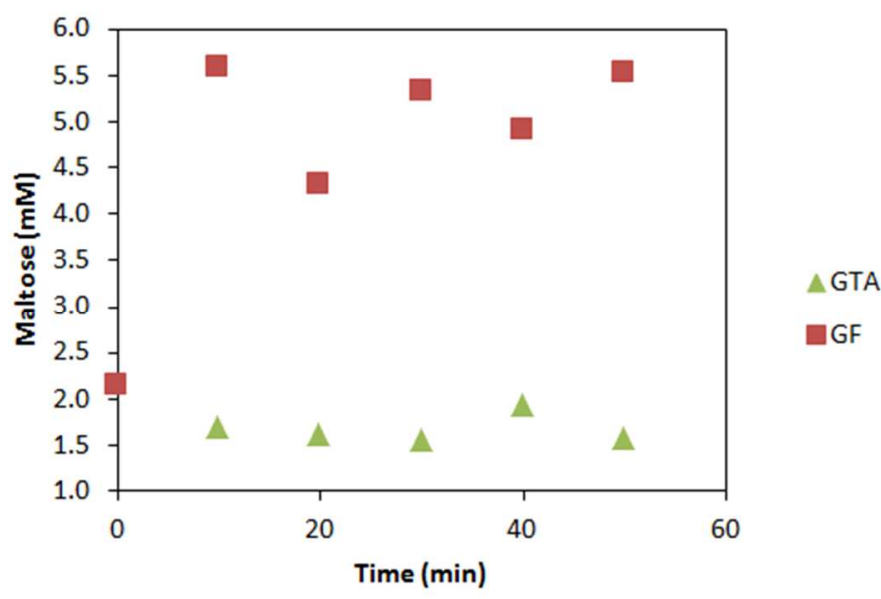

Figure S5 - Mutanase activity on GTA and GF films measured by observing the level of digestion product maltose as it reacts with 3,5-dinitrosalicylic acid stabilized with potassium sodium tartrate (Rochelle salt) to read the absorbance at $540 \mathrm{~nm}$.
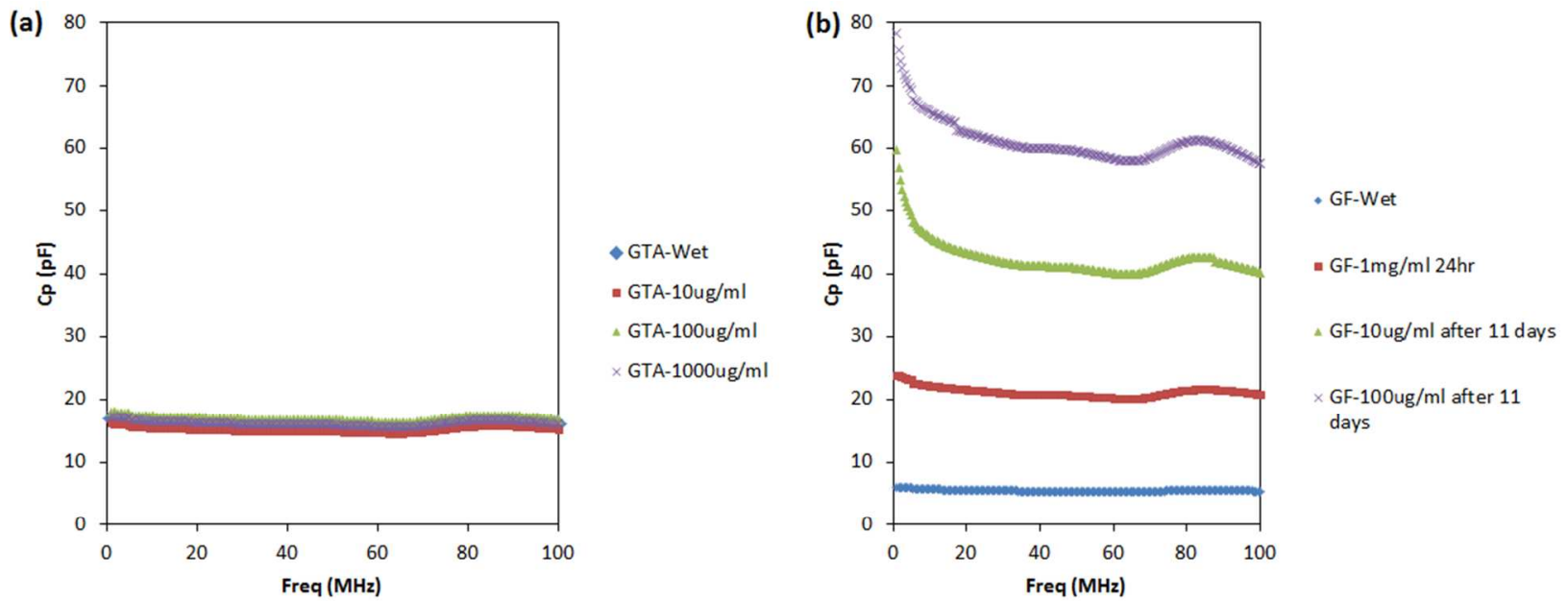

Figure S6 - Effect of mutanase digestion on capacitance of GTA (a) and GF (b) films as measured by 4 point impedance analyzer with dielectric test head. 


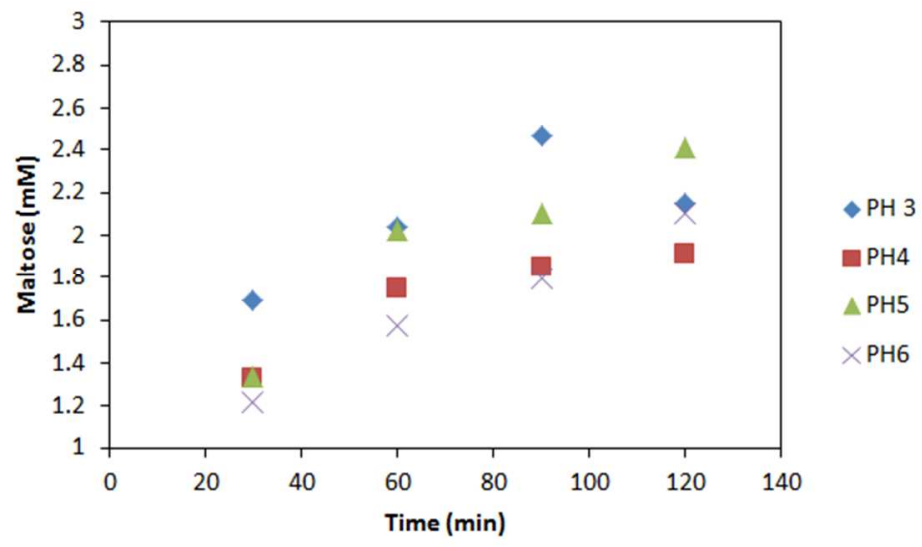

Figure S7 - Mutanase activity assay reveals broad pH tolerance.

\section{S21 VNA Setup and Matlab Code for Copper Mountain DAQ}

Two copper loops ( $5 \mathrm{~cm}$ diameter) are mutually grounded on a BNC Female Panel Mount Connector (Fig S8) and connected to the two ports of the $\$ 4048$ Copper Mountain Vector Network Analyzer. The transmitter/receiver loops and the inductive coil are both mounted on plastic rods and secured on a sliding stage to an optics table. The code below is adapted from the vendor to acquire data via Matlab.

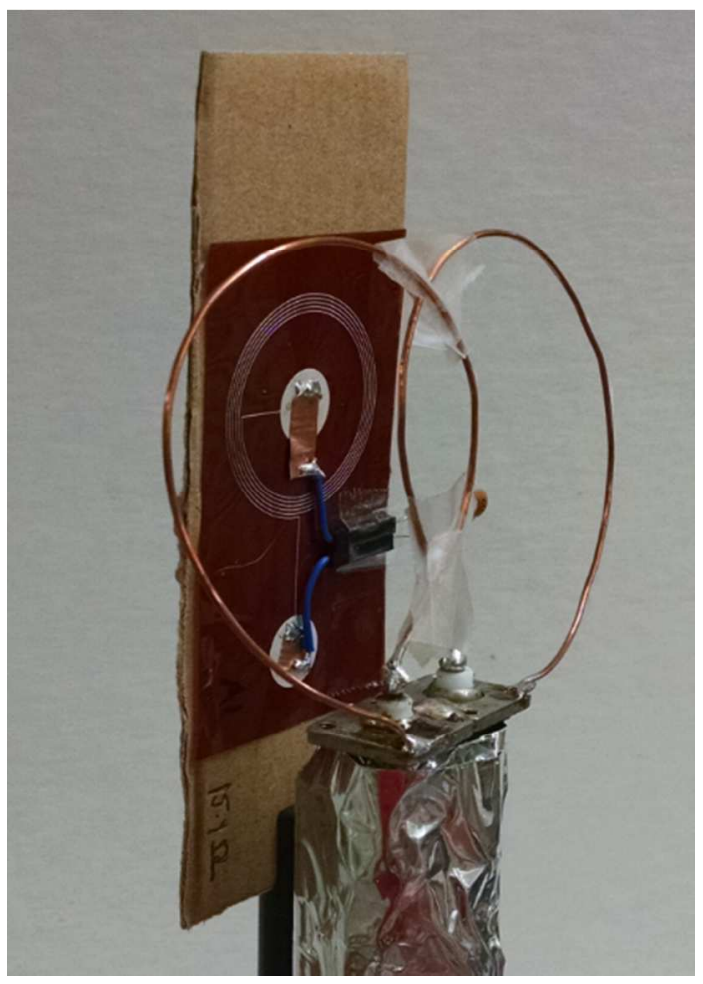




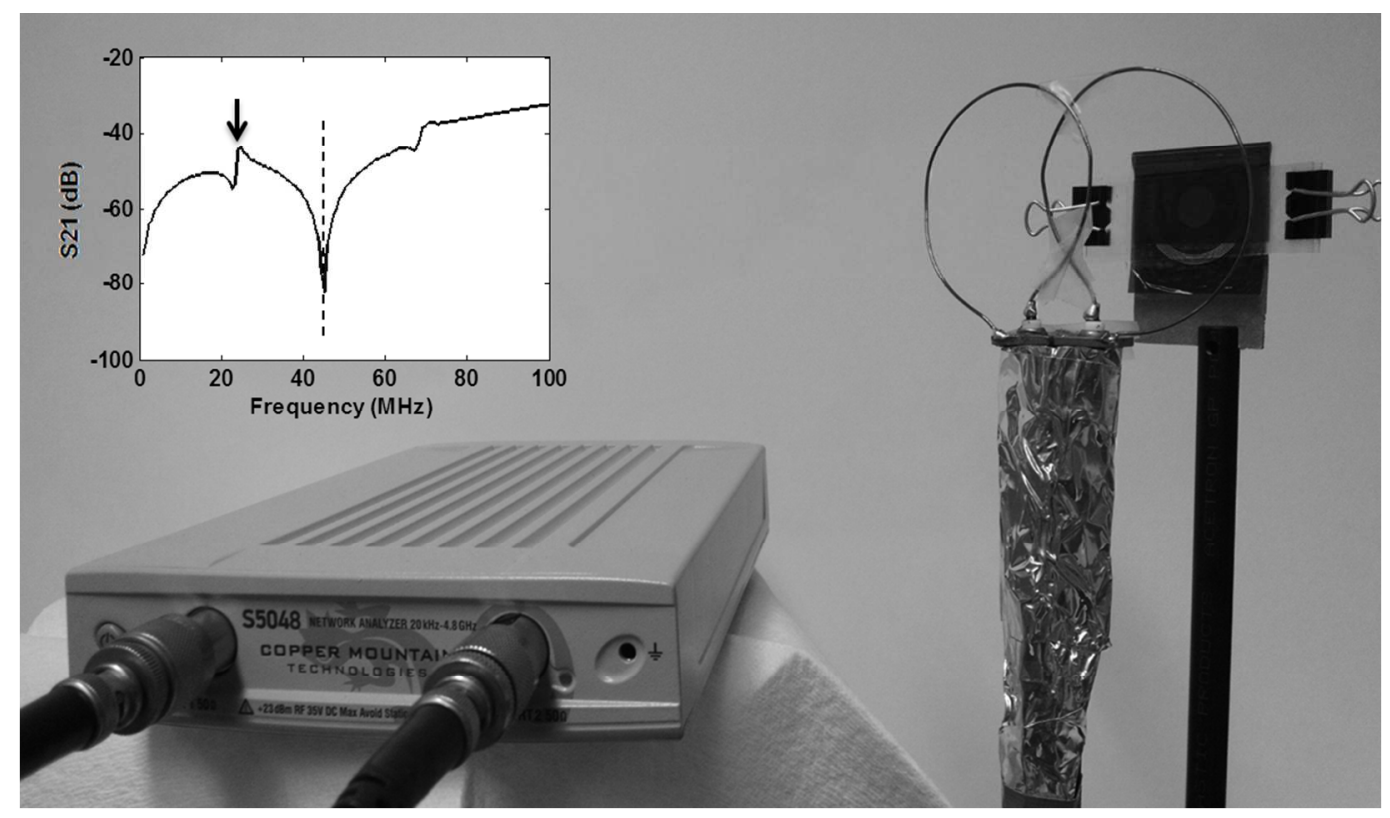

Figure S8 - Two loop antenna for S21 parameter measurement of RF 'bio-logic' sensor. In the top photo the displacement effect is being measured with a $6 \mathrm{pF}$ ceramic capacitor (see Fig 3a of main paper for results). The bottom photo shows the VNA measuring a folded device with the collagen, subtilisin, and calcium as capacitor.

\section{Code for VNA Data Acquisition}

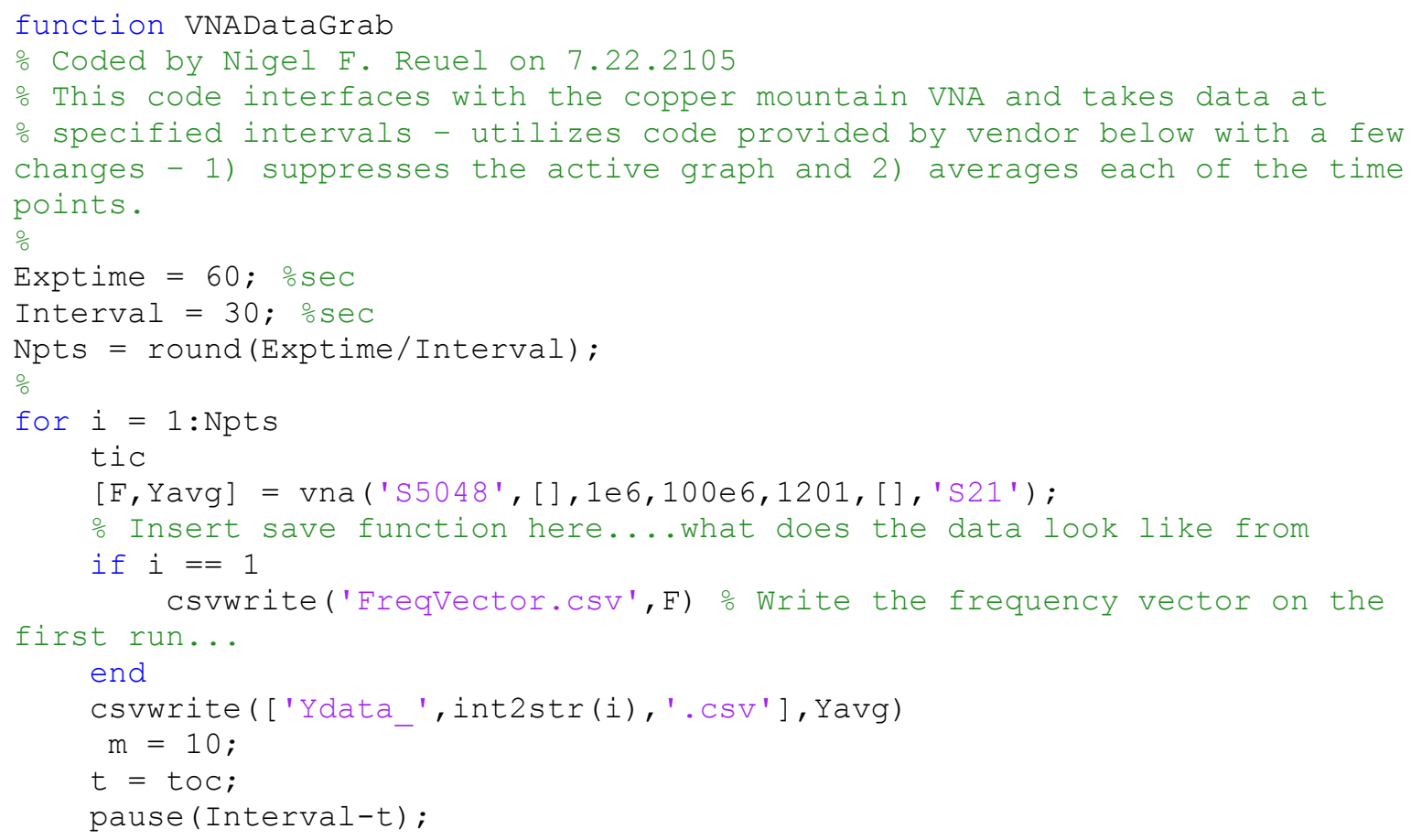




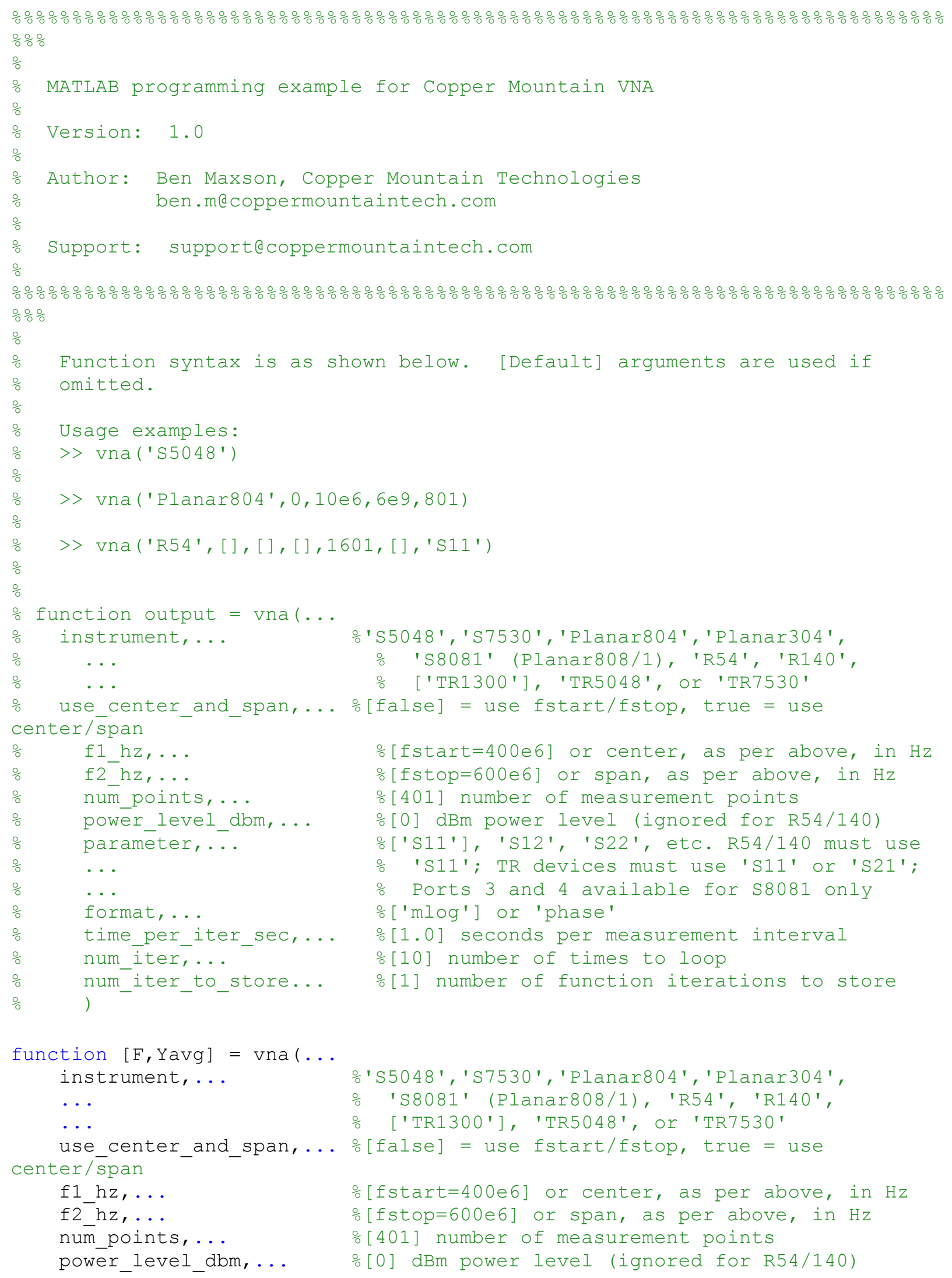




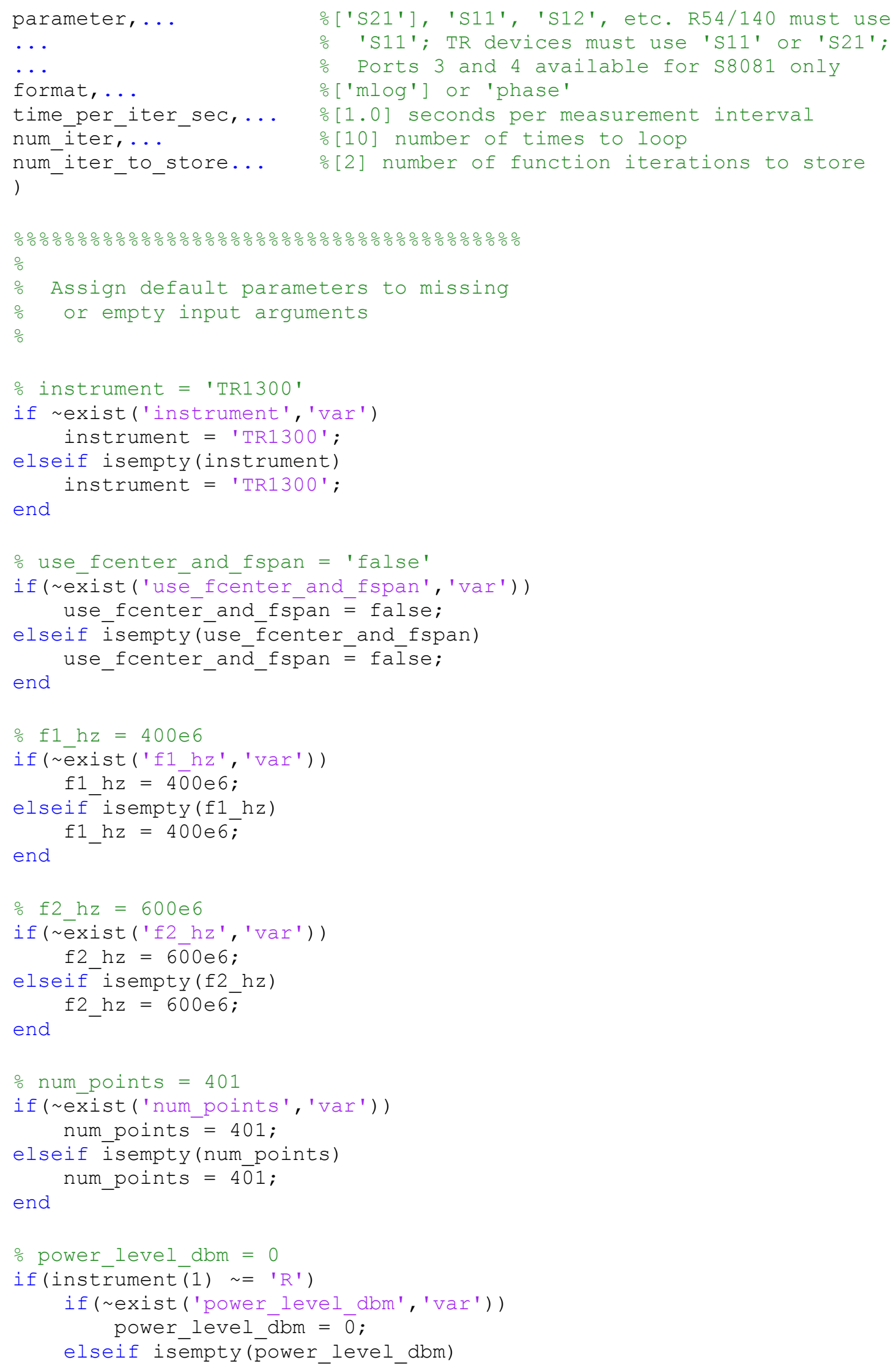




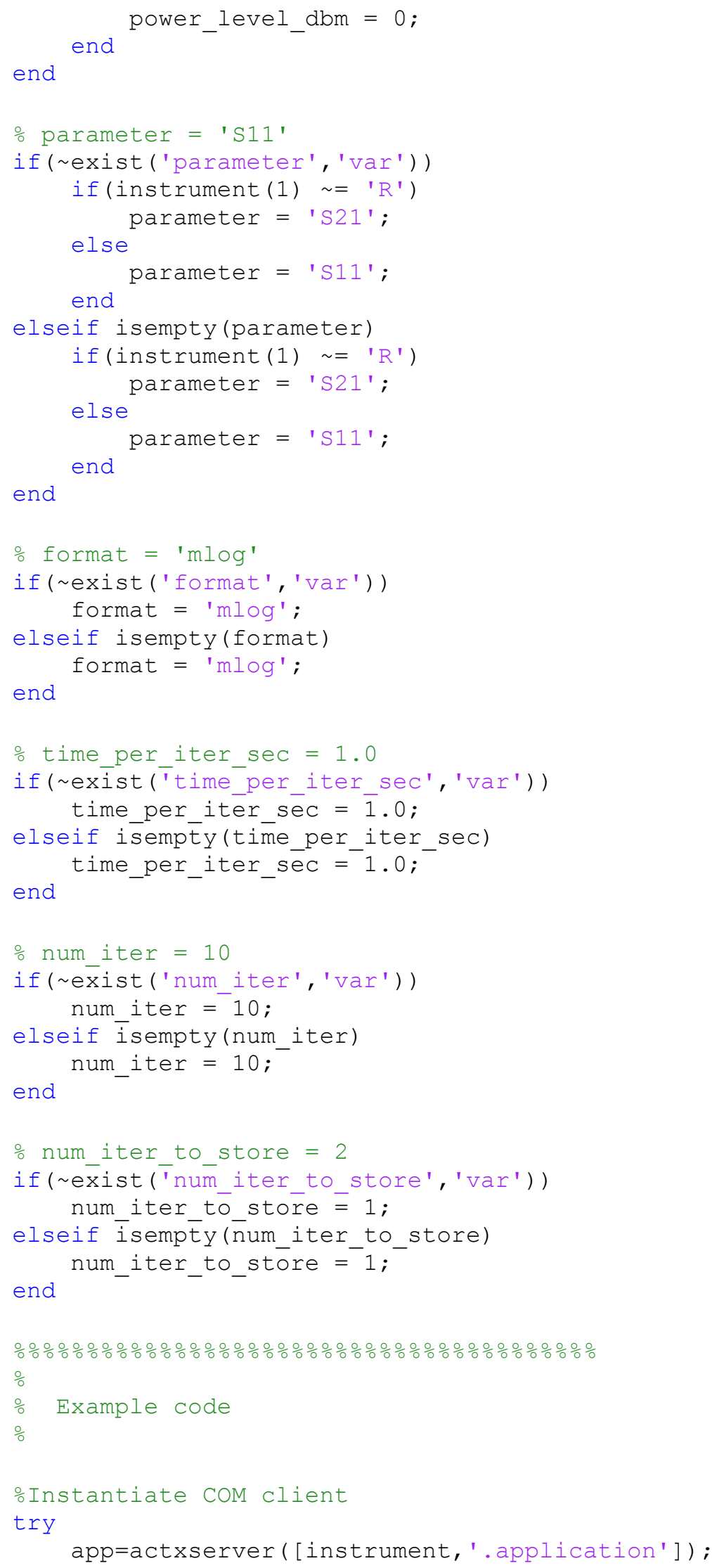




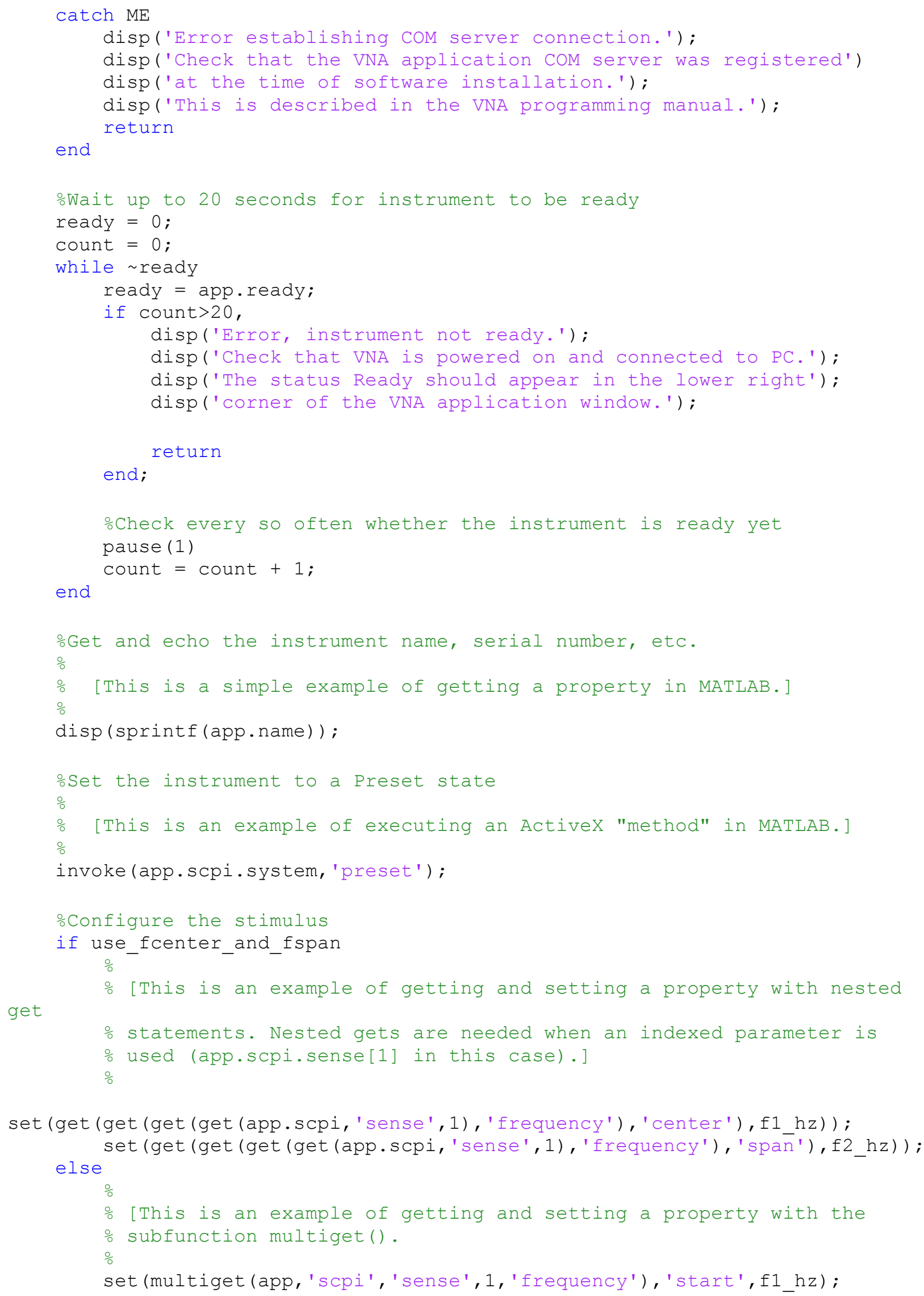




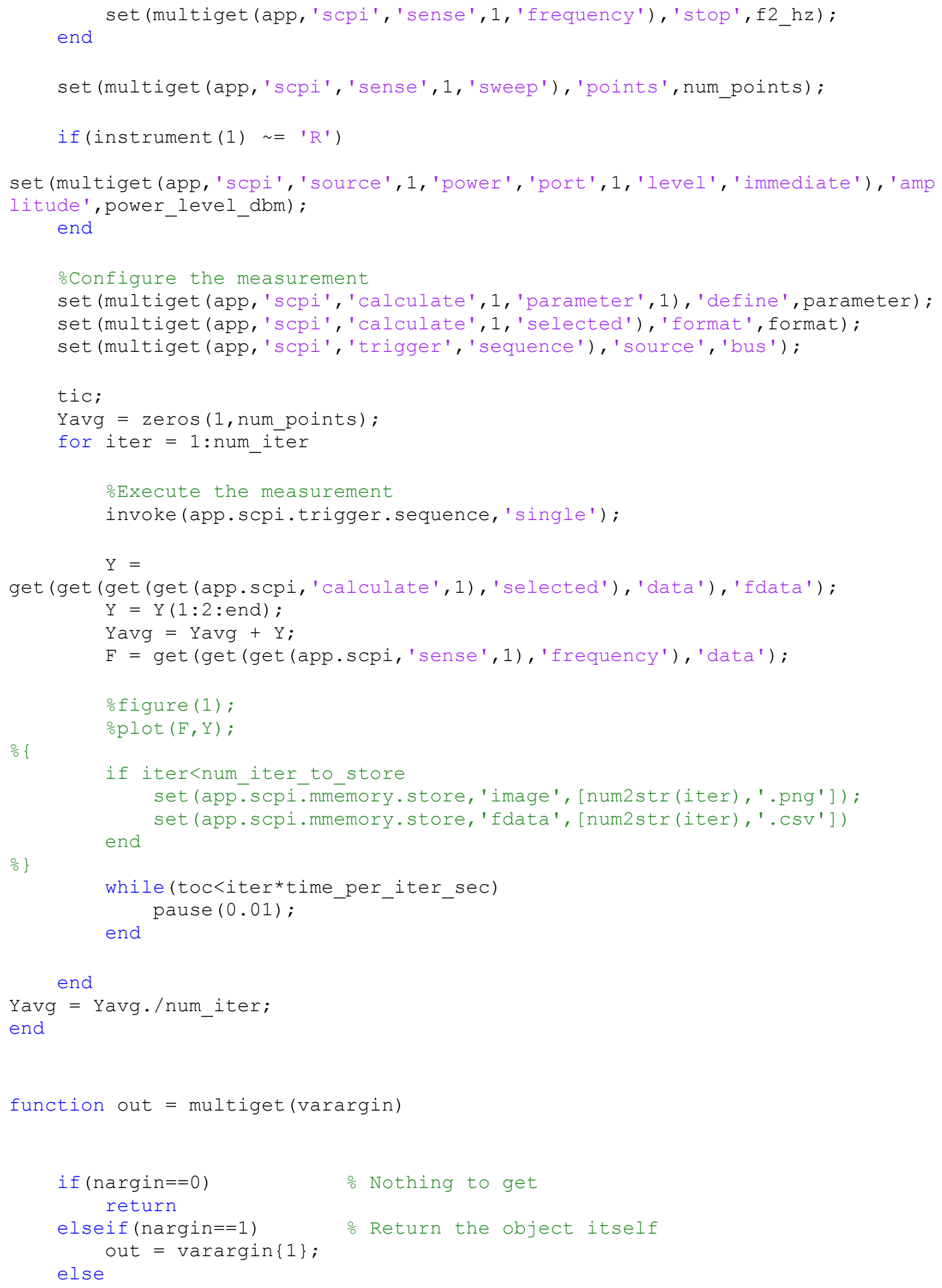




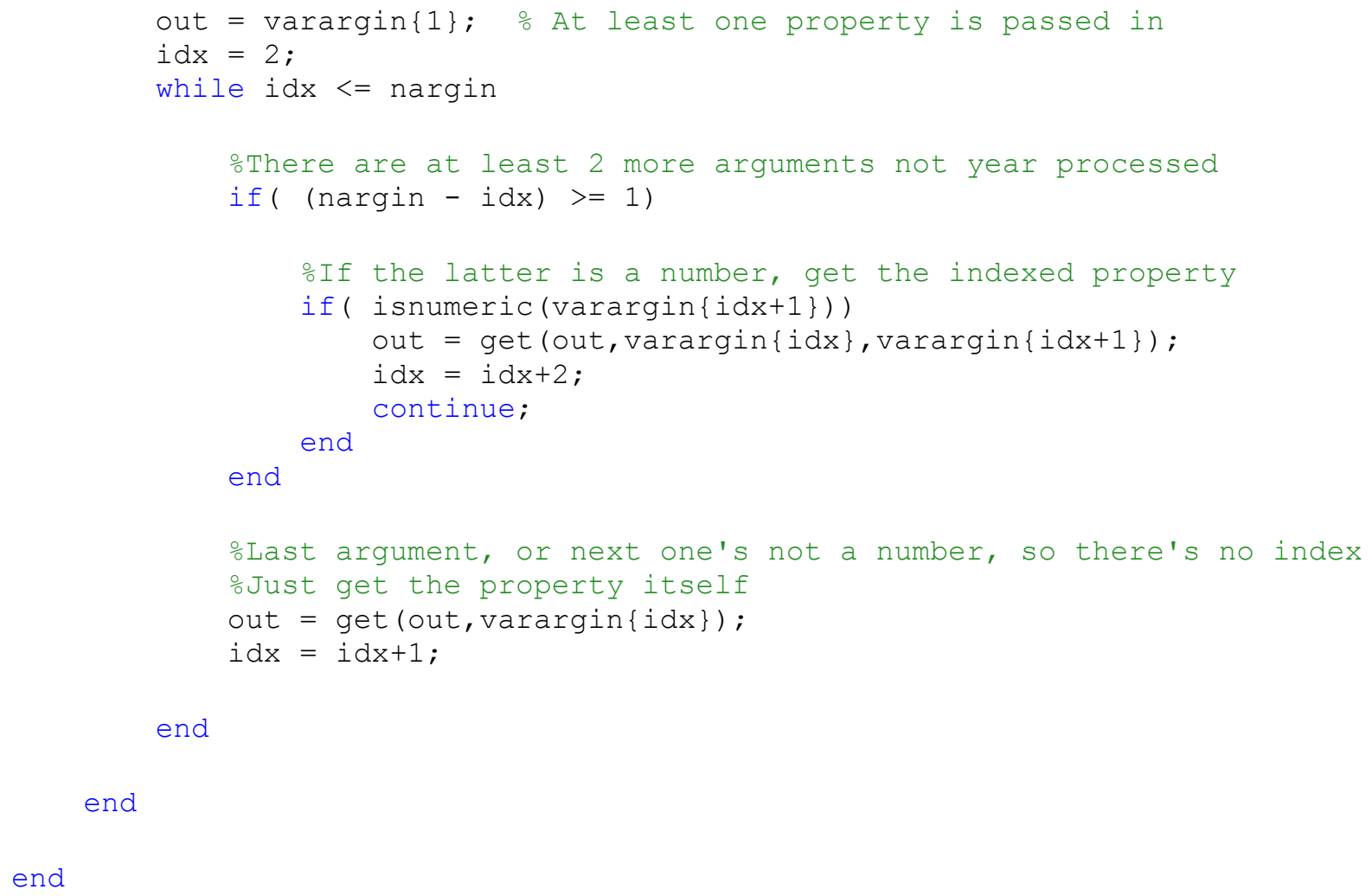

\section{Effect of Spacing at Same Frequency as Null $(40.68 \mathrm{MHz})$}

The maximum read range of the wireless sensor occurs when the resonant frequency of the device matches the null frequency valley of the two loop antenna from the VNA. This allows for a larger change in the S21 parameter to be observed. This can be achieved by either changing the substrate material thickness or composition to create the appropriate capacitance to match the device frequency to the VNA null frequency OR the VNA loop size and spacing can change to match the null frequency to the device frequency. Here we show the signal peak height vs. displacement when the two frequencies are matched (done with a ceramic capacitor $-6 \mathrm{pF}$ to match the $40.58 \mathrm{MHz}$ null frequency of the VNA) (Figure S9). The signal peak at $15 \mathrm{~cm}$ is still well above the null frequency minimum (dotted red line). 

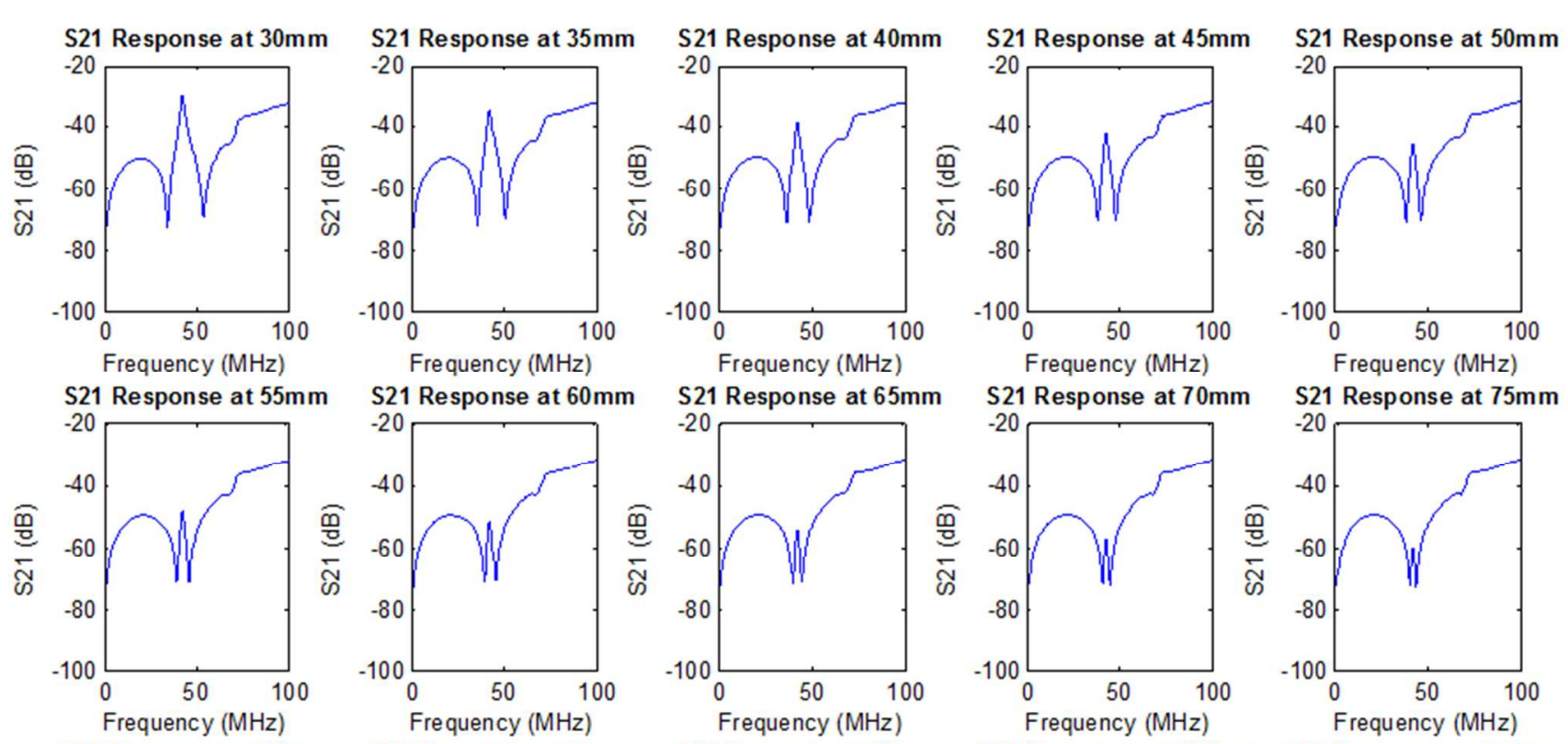

Frequency $(\mathrm{MHz})$
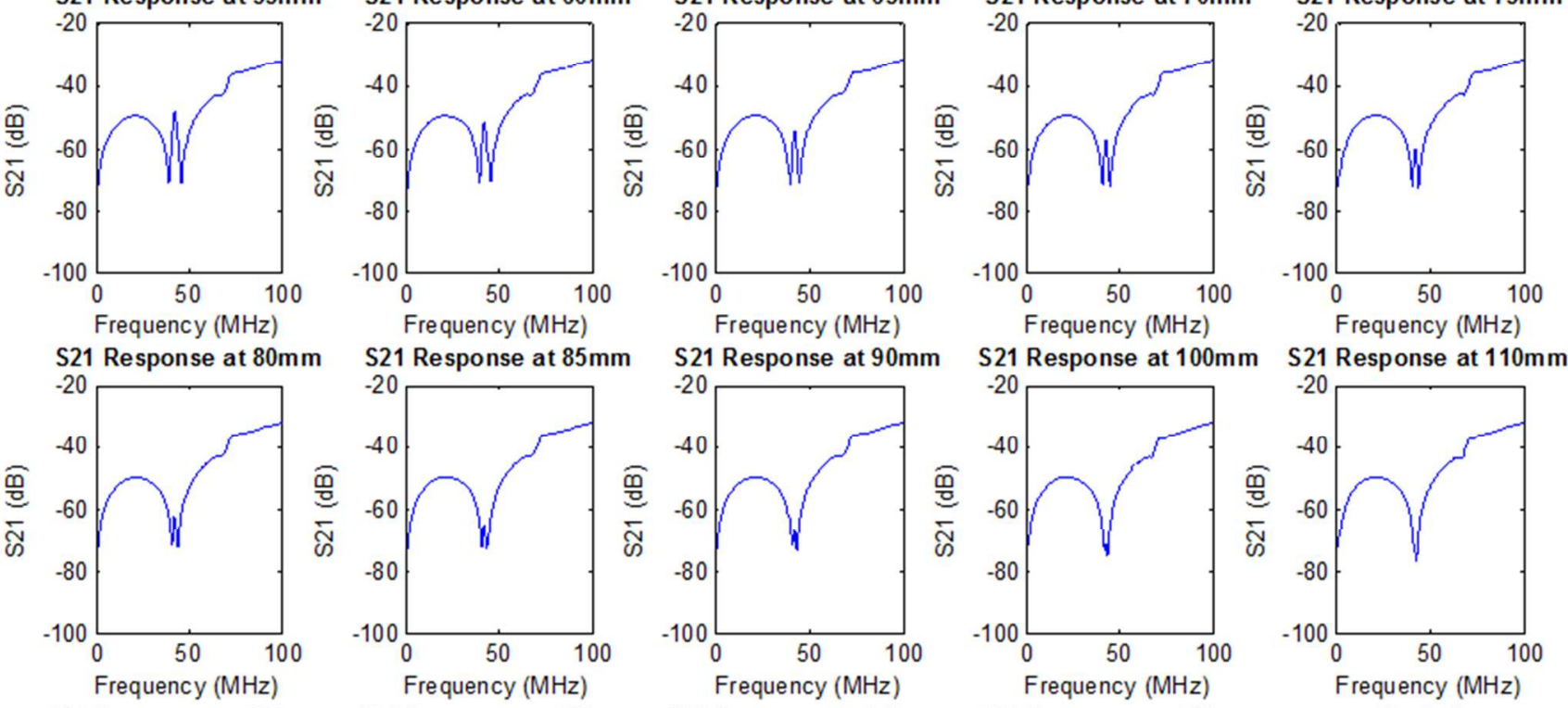

S21 Response at $90 \mathrm{~mm}$
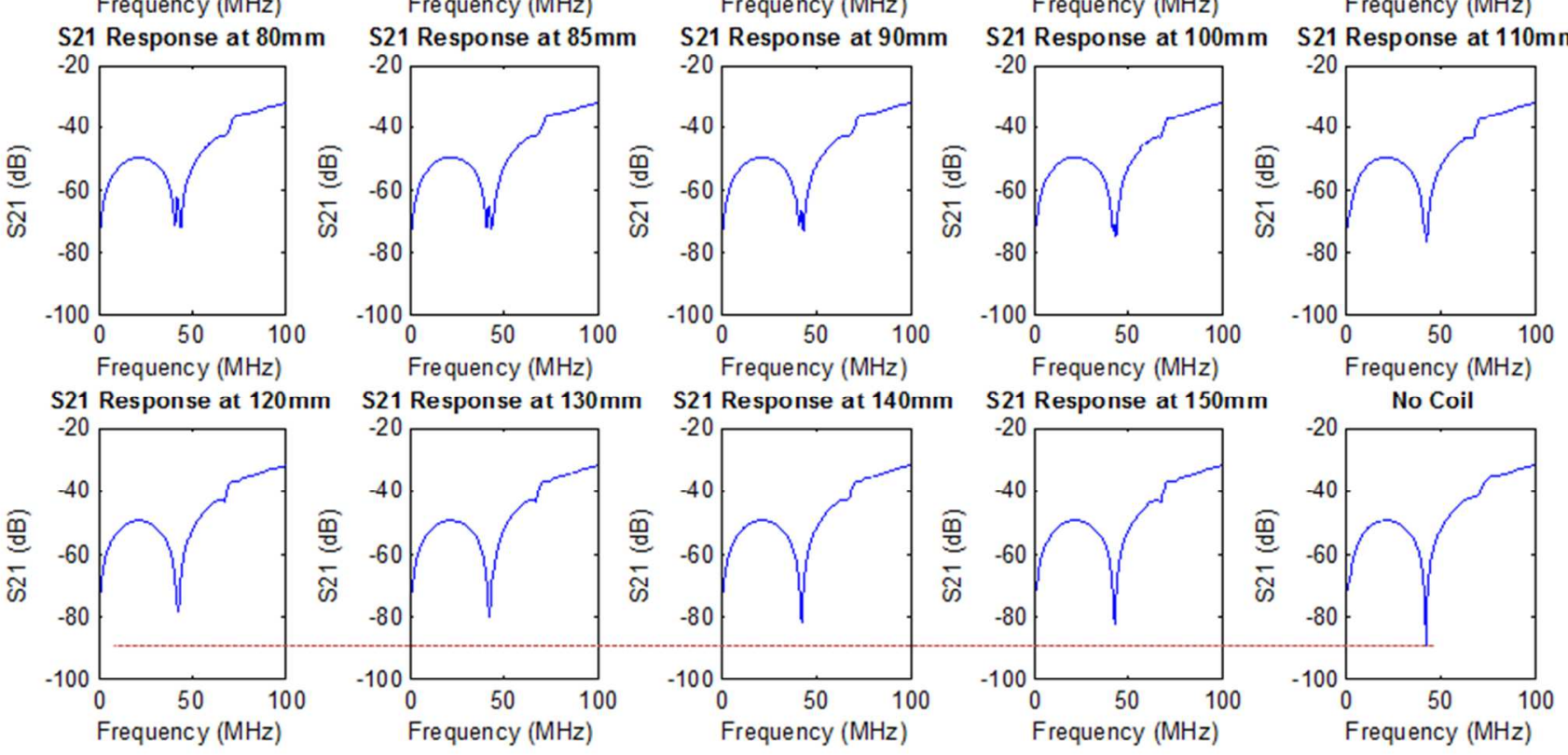

Figure $\mathbf{S 9}$ - Effect of spacing when device resonant frequency is matched to the null frequency valley. At $15 \mathrm{~cm}$, the response is still detectable above the null valley minimum when no coil is present (red dotted line)

\section{Response curves from the other divalent cations}

The full response curves for the selectivity experiments to magnesium, copper, and manganese are shown in Fig S10. The second test of the manganese (II) addition to confirm the positive shift response is shown in Fig S11. 

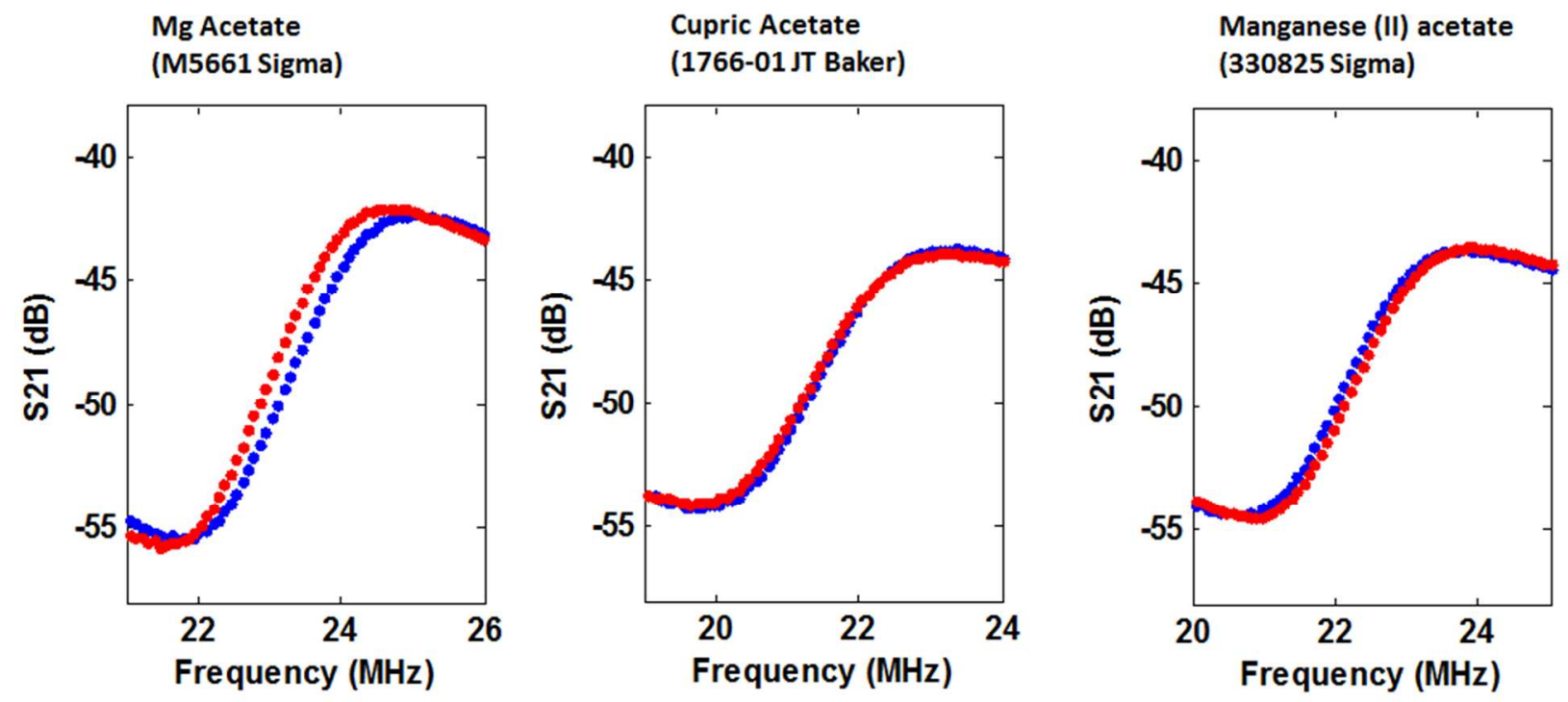

Figure $\mathbf{S 1 0}$ - Full response curves in the magnesium, copper, and manganese selectivity experiments.

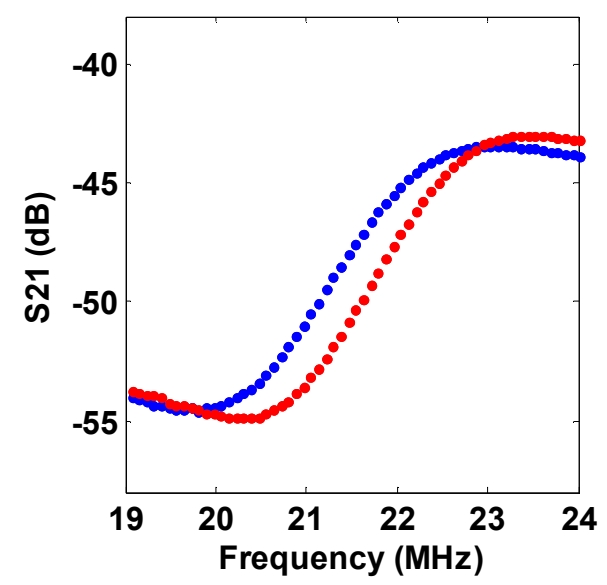

Figure S11 - Replicate of manganese experiment confirming positive shift. This test yielded a larger shift of $454 \mathrm{kHz}$.

\section{Effect of Spacing and rotation at device resonant frequency $(\sim 25 \mathrm{MHz})$}

To assess the limitations of this wireless measurement technique we observed how displacement and rotation affected the shape of the response curve. As covered in the paper, the sigmoidal response curve can be fit with a four parameter logistics curve. The span (height of the curve) and the inflection point are found to be good measures for displacement and rotation. As the device is moved from 30 $\mathrm{mm}$ to $40 \mathrm{~mm}$ we observe a linear decrease in the span and linear increase in the inflection point or resonant frequency (Fig S12). As the device is rotated from 0 to $60^{\circ}$ the trends are the same but are non-linear (Fig S13). We posit that the full shape of the response curve would enable correction for 
small changes in orientation and spacing between the device and reader antennas thus allowing for applications where fixed positions are difficult to maintain.
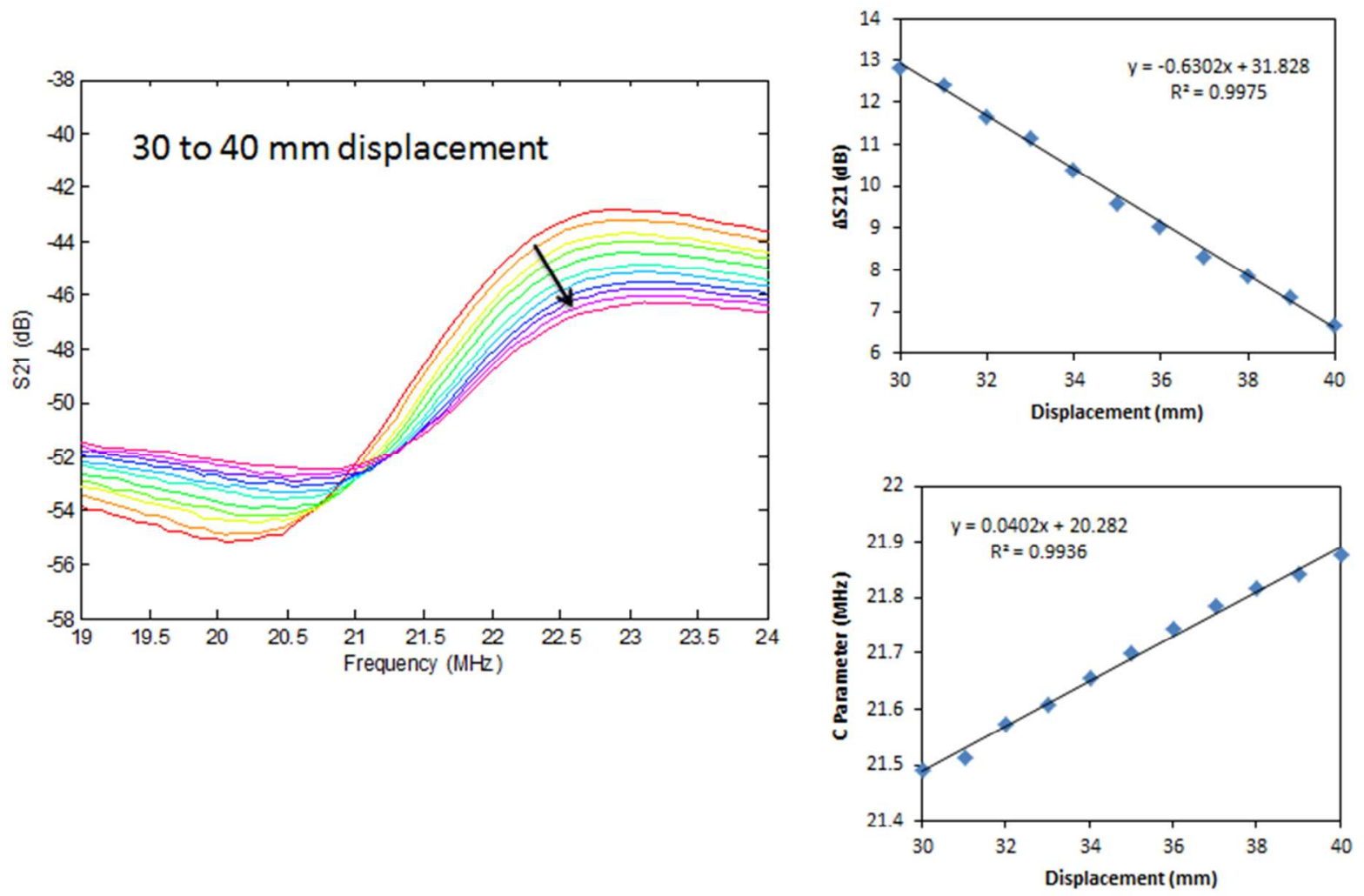

Figure S12 - The effect of spacing between device and VNA reader on response curve span (top right graph) and inflection point (C Parameter, bottom right graph). 

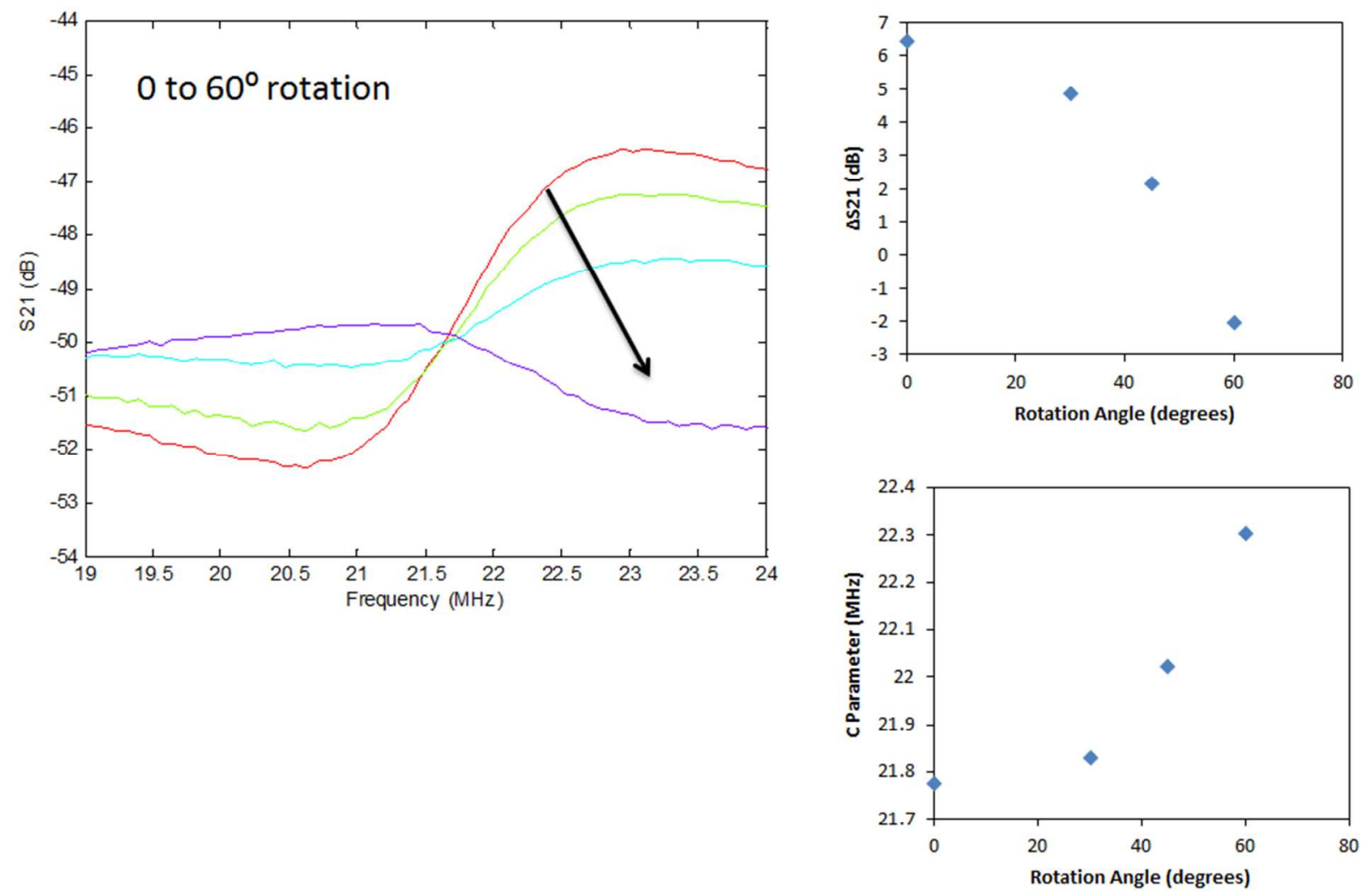

Figure S13 - The effect of orientation angle between device and VNA reader on response curve span (top right graph) and inflection point (C Parameter, bottom right graph).

\section{References}

1. Stein, E. A.; Hsiu, J.; Fischer, E. H., Alpha-Amylases as Calcium-Metalloenzymes. I. Preparation of Calcium-free Apoamylases by Chelation and Electrodialysis*. Biochemistry 1964, 3 (1), 56-61.

2. Guggenheim, B.; Muhlemann, H. R., Mutanase. Google Patents: 1982.

3. Kasat, R. B.; Paullin, J. L., Preparation of poly alpha-1,3-glucan esters and films made therefrom. Google Patents: 2014.

4. $\quad$ Caimi, P. G.; Hennessey, S. M., Increased poly ( $\alpha$ 1, 3 glucan) yield using boric acid. Google Patents: 2014. 